\title{
Philosophiques
}

\section{Précis de Sens et sensibilité. L’intentionalité en contexte}

\section{Jocelyn Benoist}

Volume 37, numéro 2, automne 2010

URI : https://id.erudit.org/iderudit/045193ar

DOI : https://doi.org/10.7202/045193ar

Aller au sommaire du numéro

Éditeur(s)

Société de philosophie du Québec

ISSN

0316-2923 (imprimé)

1492-1391 (numérique)

Découvrir la revue

Citer ce document

Benoist, J. (2010). Précis de Sens et sensibilité. L'intentionalité en contexte.

Philosophiques, 37(2), 475-478. https://doi.org/10.7202/045193ar d'utilisation que vous pouvez consulter en ligne.

https://apropos.erudit.org/fr/usagers/politique-dutilisation/ 


\section{Disputatio \\ Précis de Sens et sensibilité. L'intentionalité \\ en contexte}

JOCELYN BENOIST

Université Paris 1 Panthéon-Sorbonne

Institut Universitaire de France

Archives Husserl de Paris

Sens et sensibilité. L'intentionalité en contexte est un livre de philosophie de l'esprit. Il aborde l'esprit humain au prisme d'un concept qui a joué un rôle central dans toute la philosophie du $\mathrm{Xx}^{\mathrm{e}}$ siècle et le joue encore aujourd'hui, traversant les frontières qui séparent différentes traditions philosophiques: le concept d'intentionalité.

À ce titre sont mobilisés dans le livre des outils appartenant aux traditions phénoménologique et analytique, mais aussi, ce qui est une nouveauté dans le parcours de l'auteur, au pragmatisme.

Le concept d'intentionalité, ainsi soustrait à toute appartenance exclusive à une tradition, renvoie alors, dans sa formalité, à une caractérisation générale des actes et des attitudes de l'esprit en termes de leur orientation vers quelque chose à quoi ils sont rapportés, orientation qui les définirait dans leur singularité.

Dans la continuité du travail critique amorcé dans Les limites de l'intentionalité. Recherches phénoménologiques et analytiques ${ }^{1}$, l'auteur n'entend certainement pas rompre avec une telle représentation «intentionaliste " (c'est-à-dire faisant de l' «intentionalité » la détermination fondamentale) de l'esprit, qu'il tient en un sens pour un fait grammatical, définitionnel de ce qu'on appelle "esprit ", mais il veut, par l'analyse, en assigner plus précisément les conditions d'opérativité et donc les limites. Cette tâche critique, qui passe essentiellement par l'introduction dans l'analyse de l'intentionalité, comme structure générale du mental, d'éléments empruntés à la philosophie du langage, mais aussi à celle de l'action, ne représente cependant que la moitié de l'effort déployé, puisque le présent livre, dans ses deuxième, troisième et quatrième parties, esquisse les rudiments d'une conception alternative positive (influencée par le pragmatisme) de l'intentionalité, là où le précédent se contentait de faire fond sur une conception standard, «internaliste ", telle qu'on peut en trouver le paradigme par exemple sous des formes différentes chez Husserl et chez Searle, pour y faire des objections. Du fait de cette mutation positive du concept d'intentionalité, la critique de l'intentionalisme peut paraître en un sens moins radicale que dans le précédent

1. Paris, Vrin, 2005.

PHILOSOPHIQUES 37/2 — Automne 2010, p. 475-478 
dans ce livre qui, du reste, dans sa partie métaphysique terminale, assume pleinement l'idée d'une forme de "réalisme intentionnel».

Dans la première partie, "Perception et intentionalité », l'auteur met en évidence une limite inférieure du concept d'intentionalité, c'est-à-dire ce que la mise en jeu de celui-ci doit toujours supposer acquis, et ce sur quoi elle doit faire fond. Il s'agit de la perception que, contrairement à la position philosophique la plus répandue, l'auteur ne tient pas pour un phénomène intentionnel. Il ne s'agit pas là, de son point de vue, d'une thèse factuelle, mais d'une analyse grammaticale: la perception n'est pas non intentionnelle au sens où il lui manquerait quoi que ce soit, mais dans la mesure où cela n'a purement et simplement pas de sens de vouloir lui attribuer l'intentionalité. Lui appliquer ce genre de notion, c'est la traiter comme si on pouvait remonter en deçà d'elle et poser la question de la possibilité de ce qu'on décrit comme son "objet». Or ce qui caractérise la perception, c'est l'effectivité: il y a perception là où la chose est, de toute façon, donnée, et le problème qui peut s'énoncer en termes d'intentionalité (théorique ou morale, d'ailleurs), est alors celui de la qualification de cette chose donnée, de ce qu'on fait de la perception en question, au sens du sens qu'on lui donne. Appliquer à la perception elle-même la notion de «sens » ou de «contenu », tel que le fait couramment la tradition intentionaliste, est, de ce point de vue, un pur non-sens, qui repose sur une erreur de grammaire: sur l'ignorance du fait qu'il n'y a pas d'intentionalité qui ne soit adossée à cette présence déjà donnée des choses qui, en elle-même, n'a rien d'intentionnel. C'est ce que l'étude, d'inspiration austinienne, de notre rapport aux illusions perceptives permet d'établir.

La deuxième partie, «Pragmatique de l'intentionalité », creusant l'écart ainsi introduit entre intentionalité et présence, trouve dans le pragmatisme des ressources pour une conception dynamique de l'intentionalité, selon laquelle celle-ci n'est plus un simple fait, intrinsèque à chaque état mental, mais se construit, suivant une processualité, le «sens» intentionnel ne pouvant plus alors être inhérent à une perception par exemple, dans son détachement supposé, mais dépendant essentiellement de ce que l'agent cognitif fait de cette perception, dans l'intrigue au fil de laquelle se déploie et s'organise son expérience.

Ce modèle, qui est celui de ce que le pragmatisme appellerait « enquête » (inquiry), conduit logiquement à revenir sur la détermination originairement pratique d'une notion, l'intentionalité, dont une certaine philosophie, tout en la généralisant au delà de sa sphère d'origine (celle de l'action), a effacé certains aspects essentiels. De ce côté, il ne s'agit encore une fois pas tant de remettre en question la caractérisation générale de "l'action ", ou d'un certain type d'action, en termes d'intentionalité, que de souligner la porosité et la fragilité d'une telle détermination, porosité et fragilité qui n'en constituent pas les contraires, mais les conditions concrètes et la réalité. Le passage sur le terrain pratique, de ce point de vue, rend visible une condition 
générale de l'intentionalité: ce qu'on appellera une "contrainte de réalité ». Pas d'intentionalité qui n'ait à frayer effectivement son chemin à travers le monde, c'est-à-dire à y ajuster et négocier constamment les conditions de cette prise normative sur les choses qu'elle est. Le "projet d'action" ne représente à cet égard qu'une modalité d'intentionalité plus explicite que d'autres. Mais c'est un trait général de l'intentionalité que permet d'établir son étude.

Les troisième et quatrième parties, "Les accidents de l'intentionalité " et «Limites et réglages du contexte », qui constituent le cœur de l'ouvrage, tirent les conséquences d'une telle redétermination "pragmatique » de l'intentionalité, en interrogeant systématiquement les conditions de la référence et de la prise intentionnelles sur les choses à la lumière d'une réflexion sur les actes de langage, transposant en philosophie de l'esprit certains résultats centraux de l'analyse de type austinien. On y insiste d'abord sur la dimension de contingence qu'introduit dans toute structure de type intentionnel son inscription dans le réel et la nécessité pour elle de se construire, suivant le modèle pragmatiste suggéré dans la deuxième partie. Dans la foulée, en radicalisation des analyses esquissées dans Les limites de l'intentionalité, c'est une réflexion sur "l'intérieur» et "l'extérieur» qui est menée, au sens de ce que cela a un sens de considérer comme intérieur ou extérieur à une structure intentionnelle donnée. L'internalisme critiqué, ce n'est pas ici (ou ce n'est qu'accessoirement) celui des "pensées dans les têtes» ou des «cerveaux dans les cuves", mais l'internalisme sémantique du "contenu» qui entend déterminer purement le sens par le sens, comme si celui-ci était chose acquise en elle-même et indépendante de toute prise exercée effectivement sur le réel, avec toutes les complications afférentes, et, de façon correspondante, l'internalisme de l'acte ou de l'attitude mentale prétendant se définir par lui-même, indépendamment de tout ancrage et de tout problème de normation. Poussant jusqu'au bout le thème de la normativité de la pensée, qui est reconnue alors par lui comme le vrai nom de l'intentionalité, l'auteur interroge systématiquement les conditions d'une telle normativité, mettant en évidence pour elle, notamment dans le chapitre VIII ( Circonstances et contexte»), qui est le plus important de l'ouvrage, un double niveau de conditionalité: les conditions internes, que la pensée fixe pour ce qu'elle vise, et les conditions externes, qui la rendent possible comme telle visée, et, au sein des conditions dites externes elles-mêmes, deux dimensions: celle du contexte en tant que dispositif normatif fixé par la pensée, eu égard à sa propre possibilité, et celle des circonstances, comme ce qui tout à la fois donne un contenu concret à cette notion de contexte et peut intrinsèquement venir en perturber le fonctionnement réglé. Le thème, une fois de plus, est celui de la fragilité, mais par là-même de la réalité, des différents ordres intentionnels.

La dernière partie, "Sur la possibilité d'un réalisme intentionnel », tire la leçon métaphysique de l'ensemble. Par «réalisme intentionnel», l'auteur entend un réalisme qui fait une place à l'intentionalité, accorde l'irréductibilité 
du fait intentionnel (c'est-à-dire de l'ordre normatif en général, qu'il soit théorique ou pratique) et la valeur que celui-ci donne aux choses, mais en même temps ne place pas la réalité du réel sous le contrôle de ce fait, mais reconnait, en dernier ressort, la fondamentale indifférence du réel, qui peut toujours déjouer ou renverser tous nos ordres intentionnels. Il s'agit d'éviter les écueils symétriques d'une métaphysique purement naturaliste, qui veut court-circuiter le fait intentionnel et s'oblitère dans sa propre intentionalité, et d'une métaphysique anthropomorphique, qui projette sur le monde des prédicats intentionnels séparés des constructions normatives qui, dans les limites qu'inévitablement elles rencontrent (celles-là mêmes de la réalité), seules leur donnent sens. 